\title{
Técnicas de manejo comportamental utilizados em Odontopediatria frente ao medo e
}

\section{ansiedade}

\author{
Behavioral management techniques used in Pediatric Dentistry in the face of fear and anxiety
}

Recebido: 22/09/2021 | Revisado: 25/09/2021 | Aceito: 26/09/2021 | Publicado: 12/10/2021

\author{
Júlia Souza Moreira \\ Universidade Brasil, Brasil \\ E-mail: julia-souza74@live.com \\ Michele Cristina Silva do Vale \\ Universidade Brasil, Brasil \\ E-mail: neuromvale@gmail.com \\ Manoel Lesser Francisco Filho \\ Universidade Brasil, Brasil \\ E-mail: manoel.lesser@hotmail.com \\ Kaio Mota Navarro de Souza \\ Universidade Brasil, Brasil \\ E-mail: kaiomota2015@gmail.com \\ Sara Caroline Campos dos Santos \\ Universidade Brasil, Brasil \\ E-mail: saracaroline2508@ gmail.com \\ Irineu Gregnanin Pedron \\ Universidade Brasil, Brasil \\ E-mail: igpedron@alumni.usp.br \\ Caleb Shitsuka \\ Universidade Brasil, Brasil \\ E-mail:cashitsuka@gmail.com
}

\begin{abstract}
Resumo
A relação comportamental entre o paciente infantil e odontopediatra pode ser consideravelmente traumática, e ansiogênica, sendo por muitas vezes considerada imprevisível devido os fatores externos que podem influenciar essa relação. As técnicas de manejo comportamental são ferramentas de grande importância, com caráter preventivo, e estabilizador do paciente pediátrico. O objetivo deste trabalho foi por meio de uma revisão de literatura apresentar algumas técnicas de manejo comportamental com ênfase nas técnicas: controle de voz, falar-mostrar-fazer, distração e modelagem. Esta revisão bibliográfica da literatura avaliou artigos publicados de 2008 a 2021, relacionados ao manejo comportamental em odontopediatria e sua relação com o medo e ansiedade. Os artigos foram pesquisados nas bases de dados como a Pubmed, Bireme, SciELO, Google Scholar e LILACS. Após leitura inicial dos resumos, foram selecionados 21 artigos que foram lidos na integra e constituem o corpo bibliográfico desta revisão. Conclusão: É de suma importância que o odontopediatra domine os conhecimentos acerca dos fatores psicológicos influenciam no comportamento do paciente, respaldando então a escolha adequada de técnica de manejo comportamental, baseada na individualidade/especificidade de cada paciente.
\end{abstract}

Palavras-chave: Odontopediatria; Manejo comportamental; Psicologia.

\begin{abstract}
The behavioral relationship between pediatric patients and pediatric dentists can be considerably traumatic, and anxiogenic, being often considered unpredictable due to external factors that can influence this relationship. Behavioral management techniques are very important tools, with a preventive and stabilizing character for pediatric patients. The objective of this work was, through a literature review, to present the main behavioral management techniques with emphasis on techniques: voice control, speaking-show-doing, distraction, and modeling. This literature review evaluated articles published from 2008 to 2021, related to behavioral management in Pediatric Dentistry and its relationship with fear and anxiety. Articles were searched in databases such as Pubmed, Bireme, SciELO, Google Scholar and LILACS. After initial reading of the abstracts, 21 articles were selected that were read in full and constitute the bibliographic body of this review. Conclusion: It is extremely important that the pediatric dentist masters the knowledge about the psychological factors that influence the patient's behavior, thus supporting the appropriate choice of behavioral management technique, based on the individuality/specificity of each patient.
\end{abstract}

Keywords: Pediatric Dentistry; Behavioral management; Psychology. 


\section{Introdução}

A odontopediatria pode ser compreendida como a especialidade odontológica que tem por objetivo o estudo e cuidado do paciente pediátrico. Essa especialidade tem muitas vezes como barreira as questões de ordem psicológicas, e psicossomáticas que podem influenciar mesmo antes do paciente chegar ao consultório. Esses fatores podem influenciar diretamente desde anamnese, consulta propriamente dita, exames, terapia farmacológica, e prognóstica do paciente pediátrico. Fatores que vão desde a não colaboração, fobias, desafios de ordem psicológica, até traumas familiares que foram passados dos pais/tutores para a criança, interferindo diretamente no adequado andamento do tratamento odontológico. (Brandenburg \& Haydu, 2009; Possobom, 2007; Maltarollo et al., 2020).

A relação entre o profissional e o paciente é comumente descrita na literatura como potencialmente ansiogênica, sendo muitas vezes permeadas por comportamentos hostis, que são correlacionados com fatores de ordem psicológica e podem ser observadas tanto nas crianças como em seus tutores legais, por muitas vezes inviabilizando o adequado do tratamento, sendo fator de extrema relevância na motivação do profissional, levando a frustração, e tolerância a determinados perfis psicológicos, e comportamentais de pacientes pediátricos. (Brant, 2015; Tovo, et al, 2016, Vasconcellos et al, 2017, Shitsuka et al., 2019). Esses comportamentos tem sido comumente associados a uma necessidade de evitação, ou fuga, que geram uma desafiadora barreira para a prestação de serviços para esses pacientes. (Cardoso \& Loureiro, 2008).

A não cooperação devido a recusas ou comportamentos hostis durante o tratamento odontológico tem proporcionado uma série de debates na comunidade cientifica, na busca de protocolos e, respostas de como melhor lidar com as reações de experiências passadas que afetam a relação, profissional, e paciente tendo como base a ansiedade, medo, repulsa e estresse. Todavia, cabe ao odontopediatra conhecer e compreender as fases do desenvolvimento infantil, assim como suas manifestações comuns a cada faixa etária. Essa percepção do universo da criança permitirá ao odontopediatra, uma analise mais apurada e fidedigna do contexto geral com intuito de escolher as melhores técnicas de manejo comportamental para cada paciente. (Brandenburg \& Haydu, 2009; Brandernburg \& Marinho-Casanova, 2013; Oliveira, 2014).

A não colaboração infantil é pode ser pautada no desejo de evitar experiências dolorosas e desagradáveis, assim como externar sua vontade de não comparecer a consulta, visto que pacientes pediátricos em grande maioria não possuem direito de escolha entre comparecer ou não a consulta odontológica, sendo encaminhados por pais, ou tutores legais. (Albuquerque, 2010; Brandernburg, 2009). Dessa forma o atendimento do paciente pediátrico deve ter início muito antes da consulta propriamente dita, de fato ela deve ser iniciada no momento em que ele chega ao consultório, iniciando pela anamnese, objetivando escolher a técnica de manejo comportamental mais adequada partindo da análise da necessidade desse paciente de acordo com a especificidade e individualidade de cada caso. (Brandernburg, 2009).

O objetivo deste trabalho é por meio de uma revisão de literatura apresentar algumas técnicas de manejo comportamental do paciente infantil durante o tratamento odontológico.

\section{Metodologia}

Esta revisão bibliográfica da literatura avaliou artigos publicados de 2008 a 2021, relacionados ao manejo comportamental em Odontopediatria e sua relação com o medo e ansiedade. (Estrela, 2018) Foram selecionados artigos para leitura dos resumos nas bases de dados como a Pubmed, Bireme, SciELO e LILACS. Os critérios de exclusão foram baseados na data de publicação, e relação com o tema proposto pelos descritores. Após leitura inicial dos resumos, foram selecionados 21 artigos que foram lidos na integra e constituem o corpo bibliográfico desta revisão. 


\section{Resultados e Discussão}

\section{Medo}

$\mathrm{Na}$ atualidade muito se fala sobre a odontologia minimamente invasiva, se tratando de procedimentos no sistema estomatognático, porém, um atendimento inadequado pode causar traumas que serão grandemente invasivos em outra esfera, à psicológica.

Um problema que acomete grande parte das pessoas é o medo do tratamento odontológico, esse medo pode levar à recusa do tratamento e impactar negativamente na condição de saúde bucal e psicológica. Essa sensação se refere a uma reação emocional natural e desagradável a estímulos específicos que ocorrem em situações associadas ao tratamento odontológico. (Andrade et al., 2020)

$\mathrm{O}$ atendimento odontológico pode causar ansiedade e medo no paciente infantil pois ele expõe as crianças a uma nova experiência dentro do seu cotidiano, no qual envolve materiais e equipamentos. Podendo provocar desconforto físico e psicológico como dor, ansiedade e medo, correspondendo ao comportamento não colaborativo (Shitsuka et al., 2019)

O medo no paciente infantil pode estar associado a experiências traumáticas em tratamentos odontológicos anteriores e à sensação individual de vulnerabilidade, além do medo aos instrumentais utilizados durante o atendimento, ao sentimento de invasão corporal e à interação das crianças com pessoas estranhas. (Andrade et al., 2020)

Decorrente desse medo que pode ocorrer durante o atendimento e a pouca cooperação por parte das crianças, fez-se necessário o desenvolvimento e estabelecimento de técnicas de manejo comportamental para com esse público. Tais técnicas objetivam obter um comportamento mais cooperativo durante o tratamento odontológico. (Coelho et al., 2021).

\section{Falar-mostrar-fazer}

Tem por objetivo fazer com que o paciente pediátrico compreenda todo o procedimento odontológico que será realizado em três etapas: explicação do que será feito, seguido de demonstração por vias táteis, visuais, auditivas, e somente após compreensão do paciente, é realizado o procedimento. Ou seja, essa técnica se baseia na explicação verbal, e não verbal do procedimento que será realizado, com intuito de promover a compreensão e cooperação do paciente. Essa técnica agrega o paciente pediátrico como participante ativo do processo, visto que aumenta a relação paciente/profissional, diminuindo em muitos casos a resistência ao procedimento. (Brandenburg \& Marinho-Casanova, 2013; Sant'anna et al, 2020)

Brandenburg, e Marinho-Casanova, (2013), verificaram uma maior colaboração com a técnica fale-mostre-faça em crianças de 5 (cinco) á 7 (sete) anos de ambos os sexos, acredita-se que a maturidade cognitiva e a facilidade para compreensão tenha ajudado nos resultados, em contra partida em crianças com 2 (dois), e 3 (três), anos o nível de colaboração foi relativamente menor em comparação ao primeiro grupo. Esse resultado sugere que crianças com maior compreensão, e maturação cognitiva se adaptam melhor a esta técnica, e confirma a teoria apontada neste estudo que crianças mais novas e com menor maturidade cognitiva, tem maior dificuldade em compreender a tarefa, dificultando assim o uso desta técnica. (Brandenburg, \& Marinho-Casanova, 2013)

\section{Distração}

Essa técnica faz uso de algum fator que cause distração no paciente enquanto passa por procedimento odontológico. Tem como objetivo, distrair o paciente pediátrico, com intuito de evitar comportamentos não colaborativos que pode desencadear medo e estresse. Dessa forma o odontopediatra deverá utilizar recursos que possibilitem facilitar a distração do paciente, propiciando um ambiente com menos tensão. (Matos, et al, 2018)

Uma das estratégias mais utilizadas como ferramenta da técnica da distração é a música. Brant, (2015), utilizou a música como elemento para aplicar a técnica de distração com objetivo de auxiliar a criança a passar pelo procedimento 
odontológico de maneira menos traumática, para analisar a eficácia da técnica foram aferidos a FC (frequência cardíaca), e SpO2 (saturação de oxigênio), dos pacientes. Foram avaliadas 34 crianças de 4 (quatro) á 6 (seis) anos de idade, de ambos os sexos, sem experiência previa odontológica, com presença de duas cáries em molares decíduos, foram realizadas 3 consultas, sendo as duas últimas destinadas a realização do tratamento restaurador atraumático, a música escolhida foi a Sinfonia $40 \mathrm{em}$ sol menor K550 de Wolfgang Amadeus Mozart. Verificou-se uma diminuição da SpO2 em um dos grupos durante a intervenção com a técnica de distração mediada pela música, desta forma acredita-se que a música pode atuar como fator de relaxamento, e tranquilizante para atendimento do paciente pediátrico. (Brant, 2015; Matos, 2018)

As técnicas de distração podem ser úteis para controlar a ansiedade e o medo das crianças durante as consultas odontológicas. Não há contraindicações para o uso de técnicas de distração durante as consultas odontológicas de crianças e adolescentes. (Robertson et al., 2019)

A evidência sobre a técnica de distração ainda é baixa, porém a técnica pode ser eficaz no controle do medo e ansiedade odontológica durante o tratamento odontológico. (Prado et al., 2019)

\section{Controle da voz}

É uma técnica que possui grande eficiência entre crianças na fase pré escolar, pautada no tom da voz, assertividade no falar, confiança, e clareza no diálogo, sendo essa técnica a mais aceita entre pais, cuidadores e tutores. Para que a técnica seja realizada de forma assertiva, é necessário, que o cirurgião dentista possua habilidade para manter o diálogo com a criança. A mensagem deve ser clara, e objetiva para que a criança a compreenda, evitando muito interlocutores. (Albuquerque, 2010; Brandenburg, 2009; Matos, 2018).

\section{Modelagem}

A técnica da modelagem consiste em fazer com que a criança observe outras crianças com o comportamento mais adequado para a realização da consulta. Para isso, é necessário uma criança ou objeto como modelo. Acredita-se que a criança deve copiar o comportamento do modelo, adquirindo um comportamento mais cooperativo frente ao Odontopediatra, e a consulta odontológica. (Sant'anna et al, 2020).

É de consenso que na entre pais e Cirurgiões dentistas que existe uma hierarquia sobre a aceitação de pais, cuidadores e tutores para as técnicas de manejo comportamental para o paciente pediátrico, sendo a técnica do controle de voz a mais aceita, seguida de outras técnicas não restritivas. (Brandenburg, 2009; Brandenburg, 2009).

\section{Ensino das técnicas}

O ensino de Odontologia, presencial ou remotamente, deve transmitir essas orientações para que sejam aplicadas na prática profissional e o cirurgião dentista ou odontopediatra tenha o conhecimento de como conduzir uma consulta de forma adequada utilizando as técnicas de manejo comportamental para colaboração do paciente infantil durante o atendimento odontológico. (Valente et al., 2021; Santo, 2016; Rocha et al., 2021)

Desta forma, o profissional deve combater o medo da criança durante o atendimento odontológico, transformando-o em algo familiar e cotidiano para as crianças.

\section{Conclusão}

Para reduzir o medo odontológico, é necessário, portanto, que o cirurgião-dentista interaja de maneira específica com a criança, utilizando técnicas de manejo comportamental e conhecendo suas individualidades, para que a criança se sinta 
segura e tenha sua confiança. Levantamos ainda a necessidade de mais estudos sobre as técnicas de manejo comportamentais para o atendimento odontológico.

\section{Referências}

Albuquerque, C. M., Gouvêa, C. V. D., Moraes, R. C. M., Barros, R. N., \& Couto, C. F. (2010). Principais técnicas de controle de comportamento em Odontopediatria. Arquivos em odontologia 45(2), 110-115.

Alves, I. B. S., Granville-Garcia, A. F., Firmino, R. T., Gomes M. C., \& Costa, E. M. B. (2019). The use of audiovisual distraction eyeglasses as a resource in pediatric dental care: a case series. RGO. Revista Gaucha de Odontologia, 67, 1-7.

Andrade, N. M., Laureano, I. C. C., Farias, L., Fernandes, L. H. F., \& Cavalcanti, A. L. (2020). Medo odontológico em escolares: um estudo piloto utilizando o Children's Fear Survey Schedule - Dental Subscale. Research, Society and Development, 9(5), e26953124. https://doi.org/10.33448/rsd-v9i5.3124

Brandenburg, O. J., \& Haydu, V. B., (2009). Contribuições da análise do comportamento em odontopediatria. Revista de psicologia ciência e profissão, 29(3), 462-477.

Brandenburg, O, J., \& Marinho-Casanova, M. L., (2013). A relação mãe-criança durante o atendimento odontológico: Contribuições da análise do comportamento. Estudos de psicologia, 30(4), 629-640.

Brant, M. O. (2015). A música como estratégia de distração durante o atendimento odontológico de crianças um ensaio clínico cruzado. Programa de pós graduação UFMG.

Cardoso, C. L., \& Loureiro, S. R. (2008) Estresse e comportamento de colaboração em face do tratamento odontopediátrico. Revista de psicologia em Estudo, 13(1), 133-141.

Coelho, V. F. D., Coelho, L. V. D., \& Costa, A. M. G. (2021). Técnicas de manejo em Odontopediatria: uma revisão narrativa da literatura. Research, Society and Development, 10(11), e414101119489. https://doi.org/10.33448/rsd-v10i11.19489

Estrela, C. (2018). Metodologia Científica: Ciência, Ensino, Pesquisa. Editora Artes Médicas.

Maltarollo, T. H., Pedron, I. G., Medeiros, J. M. F., Kubo, H., Martins, J. L., \& Shitsuka, C. (2020). A erosão dentária é um problema!. Research, Society and Development, 9(3), e168932723. https://doi.org/10.33448/rsd-v9i3.2723

Matos, L. B., Ferreira, R. B., \& Vieira, L. D. S. (2018). Manejo de comportamento em crianças com ansiedade e estresse em clínica de Odontopediatria, 4(1):18-24.

Oliveira, C. C. O. (2014). Atividades lúdicas na odontopediatria: Uma breve revisão literaria. Revista Brasileira de Odontologia. 71(1), 103-7.

Prado I. M., Carcavalli L, Abreu L. G, Serra-Negra J. M, Paiva S. M, \& Martins C. C. (2019) Use of distraction techniques for the management of anxiety and fear in paediatric dental practice: A systematic review of randomized controlled trials. Int J Paediatr Dent. 29(5):650-668. 10.1111/ipd.12499.

Possobom R. F., Carrascoza, K. C., Moraes A. B. A., \& Costa Jr, A. L. (2007). O tratamento odontológico como gerador de ansiedade. Psicologia em Estudo. 12(3), 609-616.

Robertson M, Araujo M, \& Innes N. (2019) Anxiety and fear management in paediatric dentistry using distraction techniques. Evid Based Dent. 20(2):50-51. 10.1038/s41432-019-0020-y.

Rocha S. S. D., Joye C. R., \& Moreira M. M. (2020). A Educação a Distância na era digital: tipologia, variações, uso e possibilidades da educação online. RSD [Internet]. $9^{\circ}$ de abril de 2020 [citado $4^{\circ}$ de setembro de 2021];9(6):e10963390. Disponível em: https://rsdjournal.org/index.php/rsd/article/view/3390

Sant'anna, R. M. M., Silva, R.A., Silva, L. V., \& Almeida, T. F. (2020). Aspectos éticos e legais das técnicas de manejo de comportamento em odontopediatria: Uma revisão narrativa da literatura. Rev Bras Odontol Leg RBOL. 7(2), 70-80.

Santo E do E. (2016) Ensinar e aprender na Educação a Distância: um estudo exploratório na perspectiva das práticas tutoriais. RSD [Internet]. $8^{\circ}$ de dezembro de 2016 [citado $4^{\circ}$ de setembro de 2021];3(2):92-114. https://rsdjournal.org/index.php/rsd/article/view/16

Shitsuka, C., Friggi, M. N. P., \& Volpini, R. M. C. (2019). Influência dos pais sobre o comportamento infantil no atendimento odontológico. Research, Society and Development, 8(7), e43871154. https://doi.org/10.33448/rsd-v8i7.1154

Valente G. S. C, Moraes Érica B. de, Sanchez M. C O, Souza D. F. de, \& Pacheco M. C. M. D. (2020) O ensino remoto frente às exigências do contexto de pandemia: Reflexões sobre a prática docente. RSD [Internet]. $9^{\circ}$ de setembro de $2020 \quad$ [citado $4^{\circ}$ de setembro de 2021 ]; $9(9)$ :e843998153. https://rsdjournal.org/index.php/rsd/article/view/8153

Vasconcellos, C., Imparato, J. C. P., \& Rezende K. M. (2017). Motivation chart as supporting tool in pediatric dentristry. RGO. Revista Gaucha de Odontologia. 65(3), 276-281.

Tovo, M. F., Faccin, E. S., \& Vivian, A. G (2016). Psicologia e odontopediatria: Contextualização da interdisciplinaridade no Brasil. Aletheia, 49(2) ,76-88. 